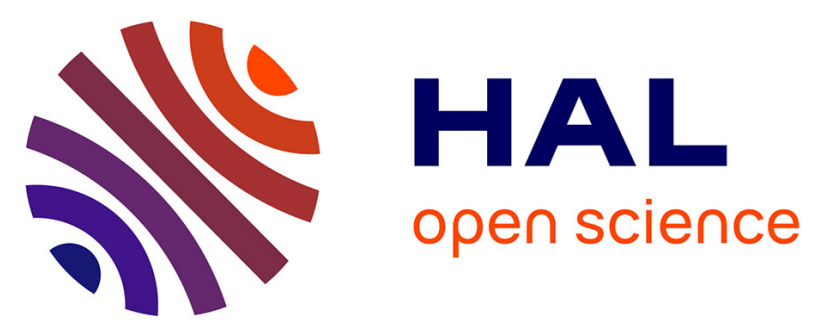

\title{
An experimental and modelling study of n-pentane oxidation in two jet-stirred reactors: The importance of pressure-dependent kinetics and new reaction pathways \\ John Bugler, Anne Rodriguez, Olivier Herbinet, Frédérique Battin-Leclerc, Casimir Togbé, Guillaume Dayma, Philippe Dagaut, Henry J Curran
}

\section{To cite this version:}

John Bugler, Anne Rodriguez, Olivier Herbinet, Frédérique Battin-Leclerc, Casimir Togbé, et al.. An experimental and modelling study of n-pentane oxidation in two jet-stirred reactors: The importance of pressure-dependent kinetics and new reaction pathways. Proceedings of the Combustion Institute, 2017, 36, pp.441 - 448. 10.1016/j.proci.2016.05.048 . hal-01464180

\author{
HAL Id: hal-01464180 \\ https://hal.science/hal-01464180
}

Submitted on 10 Feb 2017

HAL is a multi-disciplinary open access archive for the deposit and dissemination of scientific research documents, whether they are published or not. The documents may come from teaching and research institutions in France or abroad, or from public or private research centers.
L'archive ouverte pluridisciplinaire HAL, est destinée au dépôt et à la diffusion de documents scientifiques de niveau recherche, publiés ou non, émanant des établissements d'enseignement et de recherche français ou étrangers, des laboratoires publics ou privés. 


\title{
An experimental and modelling study of $\boldsymbol{n}$-pentane oxidation in two jet-stirred reactors: The importance of pressure- dependent kinetics and new reaction pathways
}

\author{
John Bugler ${ }^{1}$, Anne Rodriguez ${ }^{2}$, Olivier Herbinet ${ }^{2}$, Frédérique Battin-Leclerc ${ }^{2}$, \\ Casimir Togbé ${ }^{3}$, Guillaume Dayma ${ }^{3}$, Philippe Dagaut ${ }^{3}$, Henry J. Curran ${ }^{1}$
}

1 School of Chemistry, Combustion Chemistry Centre, National University of Ireland, Galway,
Ireland

${ }^{2}$ Laboratoire Réactions et Génie des Procédés, CNRS, Université de Lorraine, Nancy, France

${ }^{3}$ Icare, CNRS-INSIS, 1 C avenue de la Recherche Scientifique, 45071 Orléans Cedex 2, France

Published in the Proceedings of the Combustion Institute, 36, 441-448

doi:10.1016/j.proci.2016.05.048

\begin{abstract}
Two jet-stirred reactors (JSRs) coupled to gas chromatographic and spectroscopic techniques have been utilized to detect chemical species evolved during $n$-pentane oxidation at 1 and 10 atm, in the temperature range $500-1100 \mathrm{~K}$, and at equivalence ratios of $0.3-2.0$. To the authors' knowledge, this is the first study of a fuel's oxidation in two JSRs. In addition, the choice of experimental conditions results in there being the same concentration of $n$-pentane in all investigated mixtures; $1 \%$ at $1 \mathrm{~atm}$, and $0.1 \%$ at $10 \mathrm{~atm}$. This permits the additional assessment of the importance of pressure-dependent kinetics in predicting species concentration profiles. A recently published literature model Bugler et al. (2016) served as the starting point in simulating these experiments, with only minor additions and modifications necessary to achieve good overall agreement. The main adjustments were made to account for multi-oxygenated species $\left(\mathrm{C}_{5}\right.$ aldehydes, ketones, diones, etc.) detected mainly at low temperatures $(<800 \mathrm{~K})$ in both JSRs. In this paper we present new experiments, the most important of which are very well predicted using the aforementioned literature model. The effect of adding chemical pathways, which have been postulated to contribute to the generation of multi-oxygenated species, has been investigated. Finally, a brief account on the importance of pressure-dependent kinetics in the modelling of these experiments is provided.
\end{abstract}

Keywords:Jet-stirred reactor; Pentane; Kinetic modelling; CRDS; QRRK/MSC 


\section{Introduction}

In recent years there has been a renewed interest in understanding the oxidation of alkanes, with a plethora of experimental, theoretical, and modelling studies being undertaken by many research groups [1-22]. It is a welcome development that not only are these studies focussing on specific aspects which bolster our overall understanding, but many of these studies take a multifaceted approach, incorporating these different elements to "tie together" individual efforts and provide greater insights into the complex underlying processes.

This renewed interest may come as no surprise given the ubiquity of alkanes in practical combustion applications. A significant portion of petrol [23], diesel [24], and jet fuels [25] are comprised of alkanes, and their ignition properties, heat release rates, burning velocities, and chemical decomposition pathways need to be well characterised in order to improve the design of the combustors in which they are used. Not only this, but with the advent of kineticallycontrolled, low-temperature combustor technologies such as Reactivity Controlled Compression Ignition (RCCI), the chemical characterisation of fuels is now more important than ever [26].

This study represents the latest in a series of collaborative efforts to further our understanding of alkane oxidation [16-18], as well as to refine the fundamental kinetic and thermodynamic parameters which dictate the predictive capabilities of resulting chemical kinetic models. This has been largely aided by the proliferation of theoretical studies in the literature with a focus on accurate determination of these necessary model parameters. The current study builds upon these previous works by incorporating new reaction classes into an $n$-pentane mechanism with the aim of predicting multi-oxygenated species which have only recently become identifiable in experiments. The currently fashionable topic of pressure-dependent kinetics is also explored by taking new, theoretically-derived high-pressure limit rate coefficients and performing an analysis to assess their fall-off from the high-pressure limit. The resulting fall-off rate coefficients are then incorporated into the model to examine their effect.

\section{Experimental}

\subsection{Atmospheric pressure jet-stirred reactor}

The JSR experimental setup in Nancy has been used for numerous gas phase kinetic studies of hydrocarbon and oxygenated hydrocarbon fuel oxidation [27]. Experiments were performed at steady state conditions, at a pressure of $1.05 \mathrm{~atm}$, at a residence time of $2 \mathrm{~s}$, at temperatures ranging from 500 to $1100 \mathrm{~K}$, and at three equivalent ratios of $0.5,1$ and 2 with initial fuel mole fractions of $1 \%$. The reactor consists of a fused silica sphere (volume $=81.2 \mathrm{~cm}^{3}$ ) into which diluted reactant enters through an injection cross located at its centre. It is operated at constant temperature and pressure and it is preceded by an annular pre-heating zone in which the temperature of the gases is increased up to the reactor temperature before entering it. Both preheater and reactor were heated to the reaction temperature through the use of Thermocoax resistors with the temperature controlled using type K thermocouples (temperature gradients < $5 \mathrm{~K}$ ). The liquid fuel flow rate was controlled using a Coriolis flow controller, mixed with the carrier gas (helium) and evaporated in a heat exchanger. The fuel was provided by SigmaAldrich (purity > 99\%). A GC analysis of the fuel reveals a composition of $99.3 \% n$-pentane, $0.6 \%$ 
2-methylbutane, and $0.04 \%$ cyclopentane. Helium and oxygen were provided by Messer (purities of 99.99\% and 99.999\%, respectively). Products have been analyzed online using two complementary methods, namely gas chromatography (GC) and cavity ring-down spectroscopy (CRDS).

Directly connected to the outlet of the reactor, chromatographs were equipped with three columns (carbosphere packed column, PlotQ capillary column, and a HP-5 capillary column), a TCD (thermal conductivity detector) and an FID (flame ionisation detector). A GC-MS operating with electron ionisation $(70 \mathrm{eV})$ was used for products identification. Cw-CRDS, a near infrared $\left(6620 \leq \lambda \leq 6644 \mathrm{~cm}^{-1}\right.$ ) absorption spectroscopic technique was used to analyze water and formaldehyde. A tubular-glass cw-CRDS cell (length: $86 \mathrm{~cm}$, diameter: $0.8 \mathrm{~cm}$ ) working at low pressure $(1.33 \mathrm{kPa})$ was coupled to the reactor through a sonic probe. Mole fractions were calculated using two different absorption lines for each species [28].

\subsection{High pressure jet-stirred reactor}

The JSR experimental setup at Orléans has been described previously [29]. The experiments were performed at a steady state, at a constant pressure of $10 \mathrm{~atm}$, and a constant mean residence time $\tau=0.7 \mathrm{~s}$. The volume of the fused silica spherical reactor was $39 \mathrm{~cm}^{3}$. The reactants flowed constantly into the JSR and the temperature of the gases inside the reactor was increased stepwise. Uncertainties in reactor temperature, residence time, and reactor pressure are estimated as $+/-3 \mathrm{~K},+/-0.05 \mathrm{~s}$, and $+/-0.1 \mathrm{~atm}$, respectively. Prior to the injectors, they were diluted with nitrogen and mixed. A high degree of dilution $(0.1 \% \mathrm{~mol}$. of fuel) was used to minimize heat release. The reactants were high-purity oxygen $(99.995 \%$ pure) and high-purity $n$-pentane $(>99 \%$ pure from Aldrich). They were pre-heated before injection to minimize temperature gradients inside the reactor. A Shimadzu LC10 AD VP pump with an on-line degasser was used to deliver the fuel to an atomizer-vaporizer assembly maintained at $200{ }^{\circ} \mathrm{C}$. Temperature gradients of $<1 \mathrm{~K} / \mathrm{cm}$ were observed by thermocouple measurements $(0.1 \mathrm{~mm}$ Pt$\mathrm{Pt} / \mathrm{Rh}-10 \%$, located inside a thin-wall silica tube). The reacting mixtures were sampled by a movable fused silica low-pressure sonic probe and then sent to the analysers via a Teflon heated line $\left(200{ }^{\circ} \mathrm{C}\right)$. The samples were analysed online by FTIR (10 m path-length, $200 \mathrm{mbar}$, resolution of $0.5 \mathrm{~cm}^{-1}$ ) and off-line after collection and storage in $1 \mathrm{~L}$ Pyrex bulbs. Off-line analyses involved gas chromatographs (GC) equipped with capillary columns (DB-5 ms, CPSil5CB, DB-624, CP-Al203-KCl, and Carboplot-P7), a TCD (thermal conductivity detector), and an FID (flame ionisation detector). A GC-MS operating with electron ionisation $(70 \mathrm{eV}$ ) was used for products identification. For measured species, an uncertainty of $<10 \%$ was determined for concentrations higher than $10 \mathrm{ppm}$.

\section{Kinetic modelling}

\subsection{Chemical kinetic model}

A recent literature model served as the starting point for modelling of the new JSR experimental data [17]. Several reaction classes have been added based on the findings of Pelucchi et al. [14] and Ranzi et al. [15]: (i) recombination/disproportionation reactions of alkyl-peroxyl and hydroperoxyl-alkyl-peroxyl radicals, (ii) $\mathrm{H}$-atom abstractions from alkyl hydroperoxides and 
carbonyl-hydroperoxides, and (iii) decomposition of carbonyl-hydroperoxides via the Korcek mechanism [10]. These reactions and their effects on model predictions will be discussed in Section 4.2.

All simulations presented herein have utilised the perfectly-stirred reactor module in CHEMKINPRO [30]. The performance of the model against the new experimental species concentration profiles will be discussed in Section 4.1.

Rate coefficients for the reactions of $n-C_{5}$ hydroperoxyl-alkyl radicals undergoing cyclisation to cyclic ethers and hydroxyl radicals have been updated based on a recent theoretical study by Bugler et al. [22]. The pressure dependence of the rate coefficients for these reactions has been calculated via Quantum-Rice-Ramsperger-Kassel/Modified Strong Collision (QRRK/MSC) analyses [31-33].

\subsection{QRRK/MSC analyses}

The average energy transferred in a deactivating collision, $\langle\Delta \mathrm{Ed}\rangle$, is estimated with a temperature dependent function, $\langle\Delta \mathrm{Ed}\rangle=300(\mathrm{~T} / 300) 0.85 \mathrm{~cm}^{-1}$, for each of the thirteen $n-C_{5}$ cyclic ether formation reactions. Lennard-Jones parameters for the reactants are estimated to be the same as those for $n$-heptane, both containing seven "heavy" (non-hydrogen) atoms. Values for these parameters have been adopted from the work of Jasper and Miller [34], $\sigma=4.42 \AA$, $\varepsilon / k_{B}=306.39 \mathrm{~K}$. All calculations have been performed with $\mathrm{N} 2$ as the bath gas, with LennardJones parameters for $\mathrm{N}_{2}$ the same as those used by Jasper and Miller, $\sigma=3.68 \AA, \varepsilon / k_{B}=67.89 \mathrm{~K}$. The resulting rate coefficients were fitted to a modified Arrhenius form, $A \times T^{n} \times \exp \left(-E_{a} / R T\right)$, in the temperature range $500-1100 \mathrm{~K}$, in $100 \mathrm{~K}$ increments. This shortened temperature range was selected as it comfortably covers conditions where these reactions play an important role in model predictions, and also it reduces errors in fitting, resulting in a maximum deviation of $10 \%$ from the numerical data. It is expected that the QRRK/MSC approach should provide a reasonable account of the pressure dependence of these reactions based on benchmarking of the method by Pelucchi et al. [35] against the more rigorous Rice-Ramsperger-KasselMarcus/Master-Equation (RRKM/ME) approach. All rate coefficients computed via the aforementioned QRRK/MSC analyses are provided as Supplemental material in CHEMKIN format [30].

\section{Results and discussion}

\subsection{Comparisons of model simulations with experimental data}

Figure 1 shows comparisons of model simulation results with the new experimental data for a selection of the species observed in the experiments. Both 1 and $10 \mathrm{~atm}$ experimental and model-simulated species profiles are shown as a function of temperature. Figures for all species profiles as well as a detailed glossary are provided as Supplemental material. 


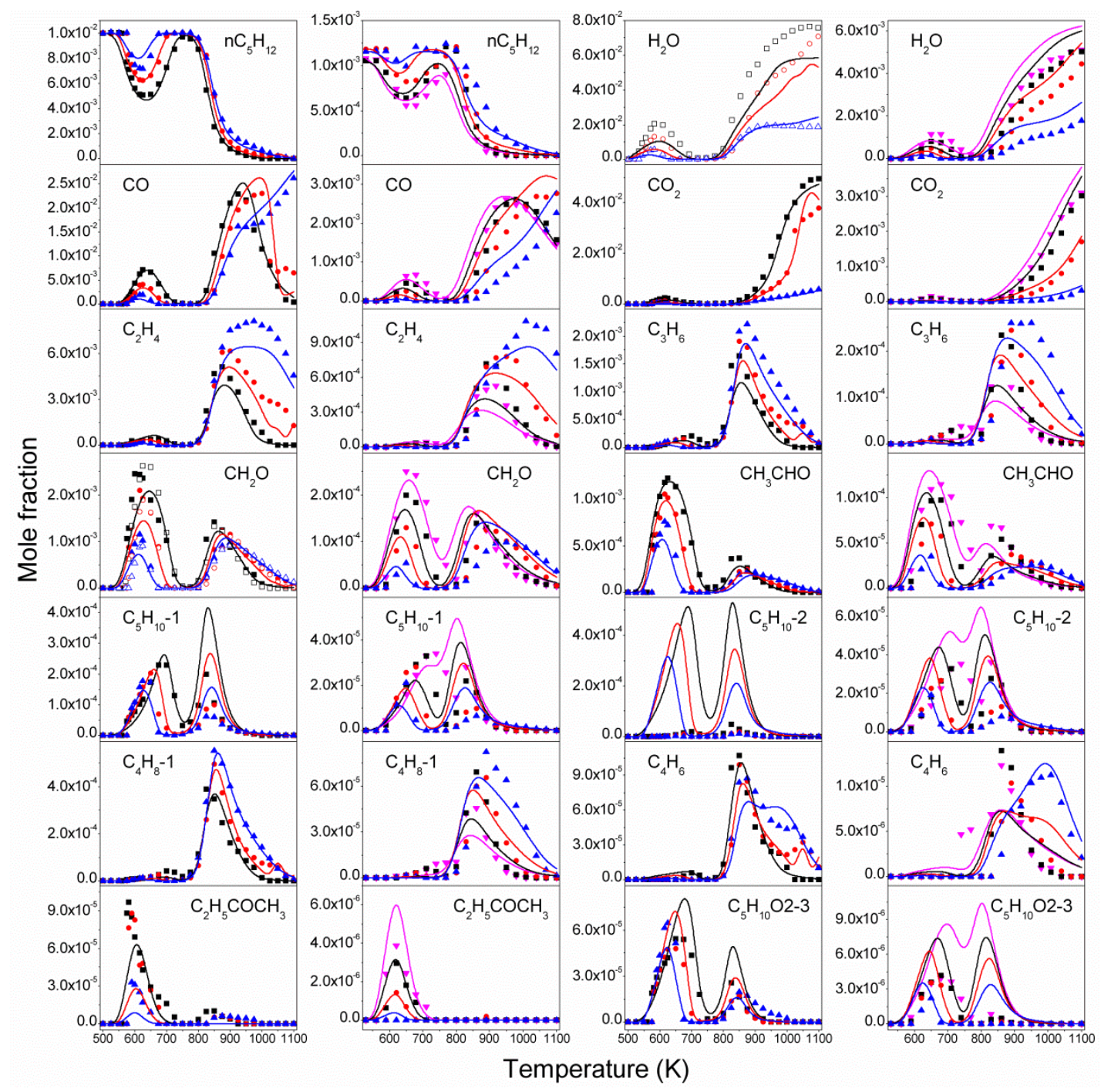

Figure 1: Model-simulated species profiles versus experimental data. The first and third columns contain $1 \mathrm{~atm}$ results, with the second and fourth columns showing $10 \mathrm{~atm}$ results. $\nabla-\varphi=0.3$, $\mathbf{-}-\varphi=0.5, \bullet-\varphi=1.0, \mathbf{\Delta}-\varphi=2.0$. Solid symbols represent species detected via GC methods, while open symbols refer to those detected via cw-CRDS.

There is excellent agreement between experimentally-observed formaldehyde concentrations at $1 \mathrm{~atm}$ detected using both GC and cw-CRDS techniques. These are the only profiles for which there exists a direct comparison of the analytical methods. For the species shown, there is generally excellent agreement between simulations and experiments. There is also approximately equal agreement between model simulations and experiments from both reactors, suggesting a consistency in the measured data. However, discrepancies exist when comparing model-simulated profiles versus experiments, most notably for the $n$-pentane, water, and 2-pentene profiles. Agreement between model simulations and experiments is generally excellent for the fuel profiles at $1 \mathrm{~atm}$, with the exception of $\varphi=2.0$ at $600 \mathrm{~K}$, where an overprediction of $n$-pentane is observed. A peculiarity arises with the fuel profiles at $10 \mathrm{~atm}$, in which slightly more $n$-pentane is measured at some temperatures compared to that detected at the lowest temperatures $(530 \mathrm{~K})$. The larger uncertainty in the reactant concentration 
measurements makes simulation of the high-pressure fuel profiles difficult, and may contribute to the poorer agreement observed when compared to the atmospheric data.

With regard to the water profiles, the model is found to under-predict concentrations at $1 \mathrm{~atm}$ across the whole temperature range, with the largest differences seen under fuel-lean and stoichiometric conditions. While the same is true of the $10 \mathrm{~atm}$ water profiles at temperatures up to $\sim 750 \mathrm{~K}$, the model then over-predicts concentrations at temperatures above this. Note that in CRDS, the uncertainties in water mole fractions are larger than $15 \%$ at high temperatures due to the huge concentration of this species in the cell which eventually leads to line saturation above $850 \mathrm{~K}$.

The final major discrepancy is in the 2-pentene profiles. Model simulations slightly over-predict 2-pentene concentrations at $10 \mathrm{~atm}$, but the over-prediction is far more extreme at atmospheric pressure. However, the relative experimentally-observed concentrations of 1- and 2-pentene are somewhat anomalous when compared at the different pressures. At 10 atm, the peak concentrations of both olefins are approximately equal across all mixture compositions. This is in stark contrast to the $1 \mathrm{~atm}$ data, where is it seen that 1-pentene is detected in peak concentrations sometimes over ten times those of 2-pentene. In the atmospheric pressure study, there is a large uncertainty on the 2-pentene measurement since in GC its peak is significantly co-eluted with the large one due to the reactant.

Despite these few oddities, the predictions of the model are mostly in close agreement with experimental observations. This bodes well for future application of the model, and may also indicate that both the overall and relative reaction rates and their equilibria, as determined by the thermochemical parameters within the model, are of adequate accuracy to predict the global and molecular characteristics of the fuel.

\subsection{Newly added reaction classes}

Of the newly added reaction classes (Section 3.1), the recombination/disproportionation reactions of alkyl-peroxyl radicals had the most significant effect on predictions of pentanal, and 2- and 3-pentanones. These reactions involve the recombination of two peroxyl radicals to produce $\mathrm{O}_{2}$ and a di-alkylperoxide, which rapidly decomposes forming a species with an alcohol group, and another containing a carbonyl moiety. For example,

$\mathrm{C}_{5} \mathrm{H}_{11} \mathrm{OO}+\mathrm{C}_{5} \mathrm{H}_{11} \mathrm{OO} \leftrightarrow \mathrm{O}_{2}+\mathrm{C}_{5} \mathrm{H}_{11} \mathrm{OOC}_{5} \mathrm{H}_{11}$

$\mathrm{C}_{5} \mathrm{H}_{11} \mathrm{OOC}_{5} \mathrm{H}_{11} \leftrightarrow \mathrm{C}_{5} \mathrm{H}_{11} \mathrm{OH}+\mathrm{C}_{4} \mathrm{H}_{9} \mathrm{CHO}$

The peroxide intermediate is not explicitly accounted for in the model due to its relative instability. Instead, these reactions of two peroxyl radicals are treated as going directly to molecular oxygen and the alcohol and carbonyl compounds. The rate coefficients used for these reactions are those proposed by Curran et al. [36] for the reactions of two alkyl-peroxyl radicals forming $\mathrm{O}_{2}$, and two alkoxyl radicals. Figure 2(a) shows model performance versus experimental data for pentanal, where it is seen that its concentrations are well reproduced by the model at lower temperatures ( 550-650 K), but are under-predicted from approximately 700-900 K. The isomerization reaction of 1-pentyl-peroxyl radical through a 4-membered transition state 
ring structure to produce pentanal and a hydroxyl radical was included to improve model predictions of the aldehyde towards higher temperatures, but this had little effect. This isomerization has a relatively high barrier ( $\sim 40 \mathrm{kcal} \mathrm{mol}-1)$, and so is effectively non-existent in the NTC temperature regime, but it is also a high frequency factor $\left(\sim 1 \times 10^{13} \mathrm{~s}^{-1}\right)$ process due to its entropic favorability. However, the latter characteristic of the reaction is not enough to overcome the former, and so does not contribute much to pentanal formation, even at higher temperatures.
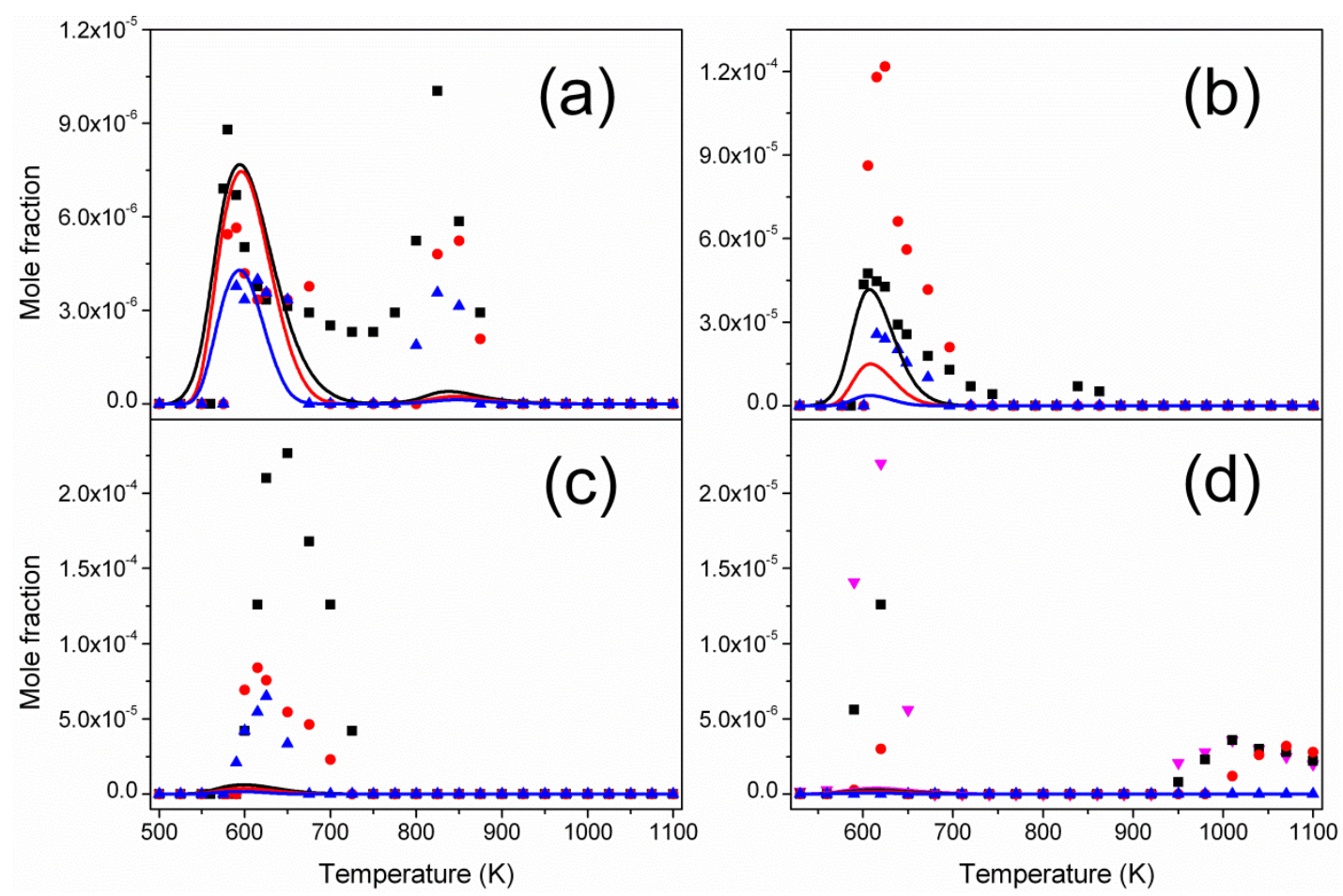

Figure 2: Model-simulated (a) pentanal, (b) acetylacetone, and (c) acetic acid profiles versus 1 atm experimental data, and (d) acetic acid profiles at 10 atm. $\boldsymbol{\nabla}-\varphi=0.3, \boldsymbol{\square}-\varphi=0.5, \bullet-\varphi=1.0$,

$\Delta-\varphi=2.0$. Dotted lines represent model-simulated acetic acid profiles upon modification to Korcek mechanism (see Section 4.2 for details).

$\mathrm{H}$-atom abstractions from alkyl hydroperoxides and carbonyl-hydroperoxides at the sites $\alpha$ - to the hydroperoxyl group were included to the mechanism, and were estimated to have a rate coefficient of $1 \times 10^{12} \mathrm{~cm}^{3} \mathrm{~mol}^{-1} \mathrm{~s}^{-1}$ per $\mathrm{H}$-atom abstracted, in close agreement with values used by Pelucchi et al. [14] and Ranzi et al. [15]. The inclusion of this reaction class had a negligible influence on the prediction of pentanal, and 2- and 3-pentanone concentrations. However, the abstractions from carbonyl-hydroperoxides results in an adequate prediction of $\mathrm{C}_{5}$ diones, though some discrepancies do exist. Although the model predicts a downward trend in acetylacetone concentrations with increasing equivalence ratio, Figure 2(b), such a trend does not present itself in the experimental data. While there is a decrease when comparing $\varphi=0.5$ to $\varphi=2.0$, unusually high concentrations of the dione are observed at stoichiometric conditions. Experimental and model-simulated peak concentrations of acetylacetone are in good agreement at fuel-lean conditions $(\varphi=0.5)$, but it is under-predicted at stoichiometric and fuel-rich conditions. 
The final class of reactions added to the mechanism is that of carbonyl-hydroperoxides undergoing unimolecular decomposition via the Korcek mechanism, resulting in the formation of organic acids. This class involves the isomerization of $\gamma$-carbonyl-hydroperoxides to cyclic peroxides, which subsequently decompose via concerted reactions to carbonyl and carboxylic acid products. The rate coefficients for these reactions are adopted from the ab initio investigations by Jalan et al. [10]. The major acid produced from the low-temperature oxidation of $n$-pentane is acetic acid, with experimental and simulated profiles plotted in Figures 2(c) and 2(d). While the model predicts some detectible concentrations of acetic acid at $600 \mathrm{~K}$ at both pressures, it is nowhere near those detected in both sets of experiments. The dotted lines in Figures 2(c) and 2(d) represent model-simulated acetic acid concentrations when the rate coefficients for isomerisation of $\gamma$-carbonyl-hydroperoxides to cyclic peroxides are increased by a factor of 10 . This change in rate coefficients has a negligible effect on the prediction of any concentration profiles other than those of organic acids (as was also observed by Pelucchi et al. [14] and Ranzi et al. [15]), but even this increase does not provide entirely satisfactory agreement with the acetic acid profiles. It may be the case that there is (at least) another pathway as yet not considered in the mechanism which may contribute to the production of acetic acid, but this pathway would have to produce multiples of the amounts found herein to be produced via the Korcek mechanism. It may also be the case that the preceding $\gamma$-carbonylhydroperoxides are not produced in high enough concentrations in the model. In either case, rectification of this discrepancy will require further investigation.

\subsection{Effect of QRRK/MSC treatment}

Figure 3 illustrates the effects of the QRRK/MSC treatment (detailed in Section 3.2) on the rate coefficient of an example cyclisation reaction of an $n$ - $C_{5}$ hydroperoxyl-alkyl radical, and the effects that this has on model-simulated concentrations of the resulting cyclic ether.
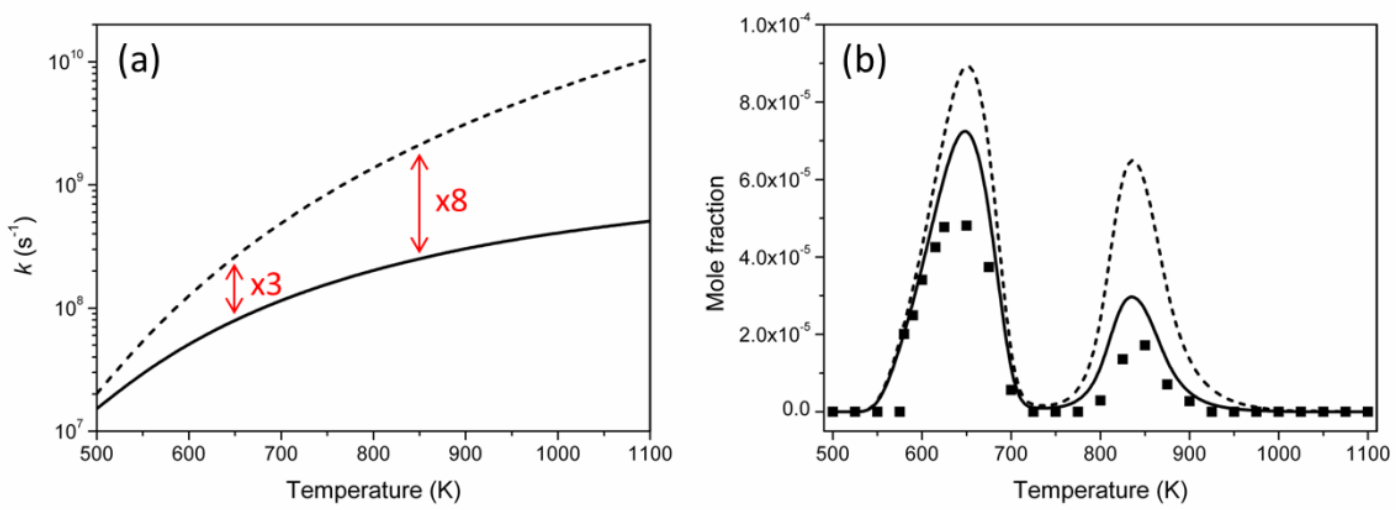

Figure 3: Effect of QRRK/MSC treatment on (a) the rate coefficient for reaction of 2-hydroperoxyl-pent-3-yl radical forming the antiperiplanar conformer of 2,3-methyl,ethyloxirane and hydroxyl radical, and (b) experimentally measured (symbols) and model simulated (lines) 2,3-methyl,ethyl-oxirane concentrations at $1 \mathrm{~atm}$, and stoichiometric conditions. Dashed lines correspond to (a) the high-pressure limit rate coefficient, and (b) model predictions using that value. Similarly, solid lines represent (a) the $1 \mathrm{~atm}$ rate coefficient, and (b) the effects of its inclusion in the model. 
There is significant improvement observed in model agreement with experimental data for the example shown in Figure 3. This example represents a maximum effect of the treatment of pressure dependence for this reaction class, as the reactions forming 3-membered cyclic ether rings have the largest rate coefficients within this class, and exhibit the most fall-off from the high-pressure limit.

Figure 3(a) shows that the difference between the high-pressure limit rate coefficient and that for $1 \mathrm{~atm}$ determined via the QRRK/MSC analysis is approximately a factor of 3 and 8 at 650 and $850 \mathrm{~K}$, respectively. The largest degree of fall-off for any of the reactions besides those forming 3-membered rings equate to factors of approximately 1.3 and 1.6 at the same temperatures and pressures as the example shown in Figure 3(a). A relatively minute amount of fuel molecules progress through pathways resulting in the formation of 3-membered cyclic ether rings, which may be counterintuitive given the high rate coefficients which typify the reactions of the hydroperoxyl-alkyl radicals which form them. This is due to the nature of the isomerisation reactions of the alkyl-peroxyl radicals which immediately precede these particular hydroperoxyl-alkyl radicals, in that they must progress through a 5-membered transition state ring, which is quite slow in comparison to alternative routes and typically represents a relatively small portion of the reaction flux $[17,19]$. This finding corroborates literature evidences $[5,8,16,17,19-21]$ that the use of high-pressure limit rate coefficients for the important lowtemperature reaction classes of alkanes is adequate under practical combustion operating conditions. For the most part this is also true in modelling atmospheric pressure environments, as shown in this study, with the treatment of pressure-dependence of uni-molecular kinetics resulting only in refinement of predictions for marginally important species.

Neither the inclusion of new reaction classes nor the treatment of pressure-dependence has had any noticeable effects on model predictions of overall reactivity, as may have been expected given the envelope of conditions over which the model had previously been validated with ignition delay time experiments, further confirming their usefulness as validation targets with which to elucidate the major reaction classes within a mechanism.

\section{Conclusions}

This study presents new jet-stirred reactor data from the oxidation of $n$-pentane at 1 and $10 \mathrm{~atm}$, in the temperature range 500-1100 K, and at equivalence ratios of 0.3-2.0. Minor modifications have been made to an existing literature model, resulting in exceptional agreement with the major species detected in both reactors.

A series of recent studies have now been conducted between several research groups on the oxidation of $n$-pentane. The model presented here can now adequately predict shock tube and rapid compression machine ignition delay times, jet-stirred reactor species concentration profiles, and laminar burning velocities.

The majority of rate coefficients for the most important reactions (especially those which control low-temperature reactivity) have been adopted from several recent quantum chemical studies, and are mostly unmodified from the values quoted in their respective manuscripts. It seems that convergence towards a consistent set of kinetic and thermodynamic values describing the oxidation of alkanes is beginning to become a reality. This, perhaps, comes as 
little surprise given the sheer amount of scientific effort and resources dedicated to the characterization of their chemical mechanisms.

Studies are now appearing quite rapidly showing application of this combined knowledge, and the relative ease with which alkane models can now predict wide ranges of experimental targets. The current fidelity of alkane models now provides an ideal base from which to probe the chemical characteristics of more exotic fuel types. Our understanding of the oxidation pathways of alkanes now allows us to more facilely elucidate the controlling factors of the oxidation of other fuels through direct comparisons.

\section{Acknowledgments}

The work at NUIG was supported by the Irish Research Council under Grant number EPSPG/2012/380. The collaboration between LRGP and NUIG was supported by COST Action 1404. The work at CNRS Orléans was supported by the ERC Advanced Researcher Grant no. 291049-2G-CSafe.

\section{Supplementary materials}

- Figures for all species profiles

- Rate coefficients computed via the aforementioned QRRK/MSC analyses

- Detailed glossary

\section{References}

[1] S. Sharma, S. Raman, W.H. Green, J. Phys. Chem. A, 114 (2010), 5689-5701

[2]F. Zhang, T.S. Dibble, J. Phys. Chem. A, 115 (2011), 655-663

[3]A. Miyoshi, J. Phys. Chem. A, 115 (2011), 3301-3325

[4]J. Zádor, S.J. Klippenstein, J.A. Miller, J. Phys. Chem. A, 115 (2011), 10218-10225

[5]S.M. Villano, L.K. Huynh, H.-H. Carstensen, A.M. Dean, J. Phys. Chem. A, 115 (2011), 1342513442

[6] A. Miyoshi, Int. J. Chem. Kinet., 44 (2012), 59-74

[7]C.F. Goldsmith, W.H. Green, S.J. Klippenstein, J. Phys. Chem. A, 116 (2012), 3325-3346

[8]S.M. Villano, L.K. Huynh, H.-H. Carstensen, A.M. Dean, J. Phys. Chem. A, 116 (2012), 5068-5089

[9]D.M.A. Karwat, S.W. Wagnon, M.S. Wooldridge, C.K. Westbrook, Combust. Flame, 160 (2013), 2693-2706 
[10] A. Jalan, I.M. Alecu, R. Meana-Panñeda, et al., J. Am. Chem. Soc., 135 (2013), 11100-11114

[11]S.M. Villano, H.-H. Carstensen, A.M. Dean, J. Phys. Chem. A, 117 (2013), 6458-6473

[12]J. Zádor, H. Huang, O. Welz, J. Zetterberg, D.L. Osborn, C.A. Taatjes, Phys. Chem. Chem. Phys., 15 (2013), 10753-10760

[13]L. Cai, H. Pitsch, Combust. Flame, 161 (2014), 405-415

[14]M. Pelucchi, M. Bissoli, C. Cavallotti, et al., Energy Fuels, 28 (2014), 7178-7193

[15]E. Ranzi, C. Cavallotti, A. Cuoci, A. Frassoldati, M. Pelucchi, T. Faravelli, Combust. Flame, 162 (2015), 1679-1691

[16]K. Zhang, C. Banyon, C. Togbé, P. Dagaut, J. Bugler, H.J. Curran, Combust. Flame, 162 (2015), 4194-4207

[17]J. Bugler, B. Marks, O. Mathieu, et al., Combust. Flame, 163 (2016), 138-156

[18]L. Cai, H. Pitsch, S.M. Sarathy, V. Raman, J. Bugler, H.J. Curran, Combust. Flame (2016), In Press

[19]J. Bugler, K.P. Somers, E.J. Silke, H.J. Curran, J. Phys. Chem. A, 119 (2015), 7510-7527

[20]M.P. Burke, C.F. Goldsmith, Y. Georgievskii, S.J. Klippenstein, Proc. Combust. Inst., 35 (2015), 205-213

[21]C.F. Goldsmith, M.P. Burke, Y. Georgievskii, S.J. Klippenstein, Proc. Combust. Inst., 35 (2015), 283-290

[22]J. Bugler, J. Power, H.J. Curran, Proc. Combust. Inst., 36 (2016) in press

[23]C. Harper, J.J. Liccione, Toxicological Profile for Gasoline, U.S. Agency for Toxic Substances and Disease Registry (1995)

[24]J.F. Risher, S.W. Rhodes, Toxicological Profile for Fuel Oils, U.S. Agency for Toxic Substances and Disease Registry (1995)

[25]J.F. Risher, P.M. Bittner, S.W. Rhodes, Toxicological Profile for JP-5 and JP-8, U. S. Agency for Toxic Substances and Disease Registry (1998)

[26] R.D. Reitz, G. Duraisamy, Prog. Energy Combust. Sci., 46 (2015), 12-71

[27]0. Herbinet, F. Battin-Leclerc, Int. J. Chem. Kinet., 46 (2014), 619-639

[28]A. Rodriguez, O. Frottier, O. Herbinet, R. Fournet, R. Bounaceur, C. Fittschen, F. BattinLeclerc, 
J. Phys. Chem. A, 119 (2015), 7905-7923

[29]P. Dagaut, M. Cathonnet, J.P. Rouan, R. Foulatier, A. Quilgars, J.C. Boettner, F. Gaillard, H. James,

J. Physics E : Instrum., 19 (1986), 207-209

[30]CHEMKIN-PRO 15101, Reaction Design, San Diego, 2010.

[31]A.M. Dean, J. Phys. Chem., 89 (1985), 4600-4608

[32]A.Y. Chang, J.W. Bozzelli, A.M. Dean, Z. Phys. Chem., 214 (2000), 1533-1568

[33]L.S. Kassel, J. Phys. Chem., 32 (1928), 225-242

[34]A.W. Jasper, J.A. Miller, Combust. Flame, 161 (2014), 101-110

[35]M. Pelucchi, K.P. Somers, K. Yasunaga, U. Burke, A. Frassoldati, E. Ranzi, H.J. Curran, T. Faravelli, Combust. Flame, 162 (2015), 265-286

[36]H.J. Curran, P. Gaffuri, W.J. Pitz, C.K. Westbrook, Combust. Flame, 129 (2002), 253-280 